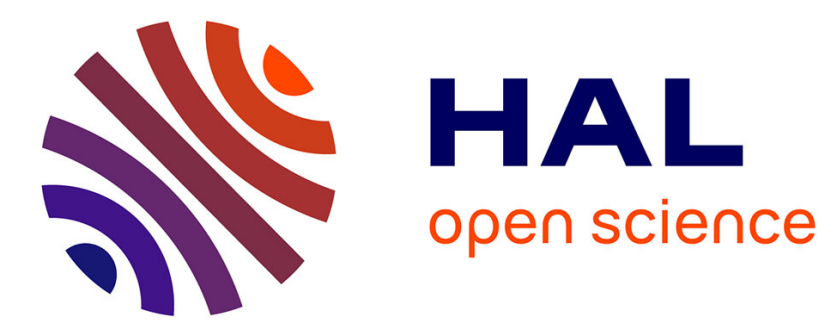

\title{
A Multiple Access Scheme for Non-Coherent SIMO Communications
}

Khac-Hoang Ngo, Alexis Decurninge, Maxime Guillaud, Sheng Yang

\section{To cite this version:}

Khac-Hoang Ngo, Alexis Decurninge, Maxime Guillaud, Sheng Yang. A Multiple Access Scheme for Non-Coherent SIMO Communications. 2018 52nd Asilomar Conference on Signals, Systems, and Computers, Oct 2018, Pacific Grove, United States. pp.1846-1850, 10.1109/ACSSC.2018.8645403 . hal-03420091

\section{HAL Id: hal-03420091 \\ https://hal-centralesupelec.archives-ouvertes.fr/hal-03420091}

Submitted on 8 Nov 2021

HAL is a multi-disciplinary open access archive for the deposit and dissemination of scientific research documents, whether they are published or not. The documents may come from teaching and research institutions in France or abroad, or from public or private research centers.
L'archive ouverte pluridisciplinaire HAL, est destinée au dépôt et à la diffusion de documents scientifiques de niveau recherche, publiés ou non, émanant des établissements d'enseignement et de recherche français ou étrangers, des laboratoires publics ou privés. 


\title{
A Multiple Access Scheme for Non-Coherent SIMO Communications
}

\author{
Khac-Hoang Ngo*†, Alexis Decurninge*, Maxime Guillaud*, Sheng Yang ${ }^{\dagger}$ \\ *Mathematical and Algorithmic Sciences Lab, Paris Research Center, Huawei Technologies, \\ 92100 Boulogne-Billancourt, France \\ Email: \{ngo.khac.hoang, alexis.decurninge, maxime.guillaud\} @huawei.com \\ †LSS, CentraleSupélec, 3 rue Joliot-Curie, 91190 Gif-sur-Yvette, France \\ Email: sheng.yang@centralesupelec.fr
}

\begin{abstract}
In this paper, we introduce a multiple access scheme for non-coherent single-input multiple-output (SIMO) communications. We first define an individual codebook for each user as the image of a non-coherent single-user codebook of smaller dimension through a bijective mapping. We investigate the available options at the decoder, including linear equalization in the absence of reference symbols, and joint multi-user decoding. In particular, we propose greedy decoders that exploit the structure of the encoder mappings to separate the signals of different users, denoise, and decode each signal as in the single-user case. Numerical results show that our scheme outperforms the timedivision multiple access and the pilot-based coherent approach.

Index Terms-non-coherent communications, multiple access, SIMO channel, Grassmannian constellations
\end{abstract}

\section{INTRODUCTION}

Channel state information (CSI) is critical to wireless communication systems over fading channels since it enables to adapt the transmission and reception to the current fading states. It is widely considered in the literature, and used in practice, to estimate the instantaneous CSI by periodically sending pilot symbols known to the receiver. The (normally imperfect) channel estimate is then used for coherent detection in the subsequent interval during which the channel is assumed to be stable [1]. This coherent approach simplifies the system design by allowing a functional split between channel estimation and data transmission-reception mechanisms, but always dedicates a fraction of communication resources to the former. Furthermore, it was shown that this approach is at a constant performance gap below the full channel capacity [2].

We consider an alternative, so-called non-coherent approach, consisting in designing the transmission and reception mechanisms without relying on the knowledge of instantaneous CSI. In block fading channel such that the fading coefficients vary independently between coherence blocks of $T \geq 2$ symbols, a representative of this approach is to transmit isotropically distributed signal belonging to the Grassmann manifold on $\mathbb{C}^{T}$ [3], [2]. Information is carried in the subspace of the transmitted signal matrix, which is not affected by the random fading coefficients. This signaling was shown to achieve the high-SNR channel capacity [2], [3]. Many Grassmannian constellation (a.k.a. codebook) designs have been proposed with different criteria, generation and decoding methods. In general, a good constellation should consist of codewords which are maximally distant from each other, thus evenly spread in the Grassmann manifold. This can be achieved by numerically optimizing the max-min distance between codewords [4], [5] However, due to the lack of structure, this kind of codebooks need to be stored at both ends and decoded with the highcomplexity maximum-likelihood decoder. A more practical approach is to impose particular structure on the codebook so as to facilitate low-complexity codebook generation and decoding while preserving good distance property [6], [7], [8].

For multi-user uplink single-input multiple-output (SIMO) communications, if the channel varies slowly, a straightforward extension of the single-user coherent approach is to let the users transmit orthogonal pilots in the first time slots and then simultaneously transmit data in the remainder of the coherence block [9]. This approach is shown to achieve the optimal degree-of-freedom (DoF) region in the two-user case [10], but only the optimal sum-DoF in general [11]. When the channel changes rapidly, some non-coherent modulation schemes have been proposed based on amplitude shift keying (ASK) [12], or differential phase shift keying (DPSK) [13], [14]. However, these studies focus rather on the massive SIMO regime and requires a large number of antennas for reasonable performance.

In this work, we consider the uplink SIMO communications in which $K$ single-antenna users transmit to a multiple-antenna receiver over block fading channel. We propose a non-coherent multiple access scheme based on Grassmannian constellations. For the encoder (in Section III), we construct for each user an individual codebook as the image of a single-user Grassmannian constellation of smaller dimension through a bijective mapping. For the receiver (in Section $I V$ ), to avoid the maximumlikelihood decoder, we propose a greedy approach exploiting the structure of the encoder mappings to separate the signals of different users without explicit channel estimation, denoise, and separately decode each signal as in the single-user case. In addition, an interference cancellation process can be performed on the decoded symbols. Simulation results (in Section $V$ ) show that our scheme outperforms the non-coherent time-division multiple access and the pilot-based coherent approach.

Notations: Random quantities are denoted with non-italic letters: normal fonts, e.g., x, for scalars; bold fonts, e.g., v, for vectors; and bold and sans serif fonts, e.g., $\mathbf{M}$, for matrices. Deterministic quantities are denoted with italic letters, e.g., 
a scalar $x$, a vector $v$, and a matrix $\boldsymbol{M}$. The Euclidean and Frobenius norms are denoted by $\|\boldsymbol{v}\|$ and $\|\boldsymbol{M}\|_{F}$, respectively. The trace, conjugate, transpose, and conjugated transpose of $\boldsymbol{M}$ are denoted $\operatorname{tr}\{\boldsymbol{M}\}, \boldsymbol{M}^{*}, \boldsymbol{M}^{\top}$ and $\boldsymbol{M}^{\mathrm{H}}$, respectively. $\boldsymbol{e}_{i}$ is the $T \times 1$ canonical basis vector with 1 at position $i$ and 0 elsewhere. $\bigoplus$ and $\prod$ denote the direct sum and Cartesian product respectively. The Grassmann manifold $G\left(\mathbb{C}^{T}, n\right)$ is defined as the space of $n$-dimensional subspaces in $\mathbb{C}^{T}$. We use a vector $x \in \mathbb{C}^{T}$ of unit Euclidean norm $(\|x\|=1)$ to represents the set $\{\lambda \boldsymbol{x}, \lambda \in \mathbb{C}\}$, which is a point in $G\left(\mathbb{C}^{T}, 1\right)$. The chordal distance between two Grassmannian lines represented by $\boldsymbol{x}_{1}$ and $\boldsymbol{x}_{2}$ is $d\left(\boldsymbol{x}_{1}, \boldsymbol{x}_{2}\right)=\sqrt{1-\left|\boldsymbol{x}_{1}^{\mathrm{H}} \boldsymbol{x}_{2}\right|^{2}}$.

\section{System MODEL}

We consider the uplink SIMO communications in which $K$ single-antenna users transmit to a receiver with $M$ antennas. We assume that the channel between the receiver and user $k$ is flat and block fading with equal-length and synchronous (across the users) coherence interval of $T$ symbol periods. That is, the channel vectors $\mathbf{h}_{k} \in \mathbb{C}^{M \times 1}, k=1, \ldots, K$, which follows an arbitrary distribution, remain constant within each coherence block of $T>1$ symbols, and vary independently between blocks. We consider non-coherent communications, i.e., $\mathbf{h}_{k}$ is unknown to both the receiver and users. Within a coherence block, each user $k$ sends a signal vector $\mathbf{x}_{k} \in \mathbb{C}^{T}$, and the receiver receives a $T \times M$ signal matrix

$$
\mathbf{Y}=\sum_{k=1}^{K} \mathbf{x}_{k} \mathbf{h}_{k}^{\top}+\mathbf{W}=\mathbf{X} \mathbf{H}^{\top}+\mathbf{W}
$$

where $\mathbf{X}=\left[\begin{array}{lll}\mathbf{x}_{1} & \ldots & \mathbf{x}_{k}\end{array}\right] \in \mathbb{C}^{T \times K}$ and $\mathbf{H}=\left[\begin{array}{lll}\mathbf{h}_{1} & \ldots & \mathbf{h}_{K}\end{array}\right] \in$ $\mathbb{C}^{M \times K}$ are the concatenation of transmitted signals and channel vectors, respectively, and we omitted the block index for simplicity. $\mathbf{W}$ is the Gaussian noise with independent and identically distributed (i.i.d.) $\mathcal{C N}\left(0, \sigma^{2}\right)$ entries. We assume that the input signal has average unit norm, i.e. $\mathbb{E}\left[\left\|\mathbf{x}_{k}\right\|^{2}\right]=$ $1, k=1, \ldots, K$. Under this normalization, the signal-to-noise ratio (SNR) of each transmitted signal at each receive antenna is $1 / \sigma^{2}$. Each transmitted signal $\mathbf{x}_{k}$ is issued from a finite individual codebook $\mathcal{C}_{k}$ of user $k$. Let $B_{k}=\log _{2}\left|\mathcal{C}_{k}\right|$ be the number of bits per codeword for user $k$.

\section{Multiple-Access Encoder Design}

Each of the individual codebooks $\mathcal{C}_{k}=\left\{\boldsymbol{c}_{1}^{(k)}, \ldots, \boldsymbol{c}_{2^{B_{k}}}^{(k)}\right\}$, $k=1, \ldots, K$, should have an unique signature so as to facilitate the receiver in separating the signals transmitted from different users. On the other hand, these signatures occupy some degrees of freedom for communications. In our design, in order to exploit the maximum degree of freedom for communications while limiting the inter-user interference, we assume $T>K$ and we construct $\mathcal{C}_{k}$ as the image of a Grassmannian codebook $\mathcal{D}_{k}=\left\{\boldsymbol{d}_{1}^{(k)}, \ldots, \boldsymbol{d}_{2^{B} k}^{(k)}\right\}$ in $G\left(\mathbb{C}^{T-K+1}, 1\right)$ through a mapping

$$
\boldsymbol{f}_{k}:\left\{\begin{aligned}
\mathbb{C}^{T-K+1} & \rightarrow \mathbb{C}^{T} \\
\boldsymbol{d}_{i}^{(k)} & \mapsto \boldsymbol{c}_{i}^{(k)}
\end{aligned}\right.
$$

Then $\boldsymbol{c}_{i}^{(k)}=\boldsymbol{f}_{k}\left(\boldsymbol{d}_{i}^{(k)}\right), i=1, \ldots, 2^{B_{k}}$. In other words, for any transmitted codeword $\mathbf{x}_{k}$ in $\mathcal{C}_{k}$, there exists a codeword $\tilde{\mathbf{x}}_{k} \in \mathcal{D}_{k}$ such that $\mathbf{x}_{k}=\boldsymbol{f}_{k}\left(\tilde{\mathbf{x}}_{k}\right)$. While the codebooks $\mathcal{D}_{k}$ can be identical or different amongst the users, the encoder mapping $f_{k}$ needs to be unique for each user. In this way, we embed the users's signatures into these encoder mappings $\boldsymbol{f}_{k}$.

In particular, we consider a mapping consisting of a linear transformation followed by a normalization, such that $f_{k}$ can be defined through a full-rank precoder $U_{k} \in \mathbb{C}^{T \times(T-K+1)}$ as

$$
\boldsymbol{c}_{i}^{(k)}=\boldsymbol{f}_{k}\left(\boldsymbol{d}_{i}^{(k)}\right)=\frac{\boldsymbol{U}_{k} \boldsymbol{d}_{i}^{(k)}}{\left\|\boldsymbol{U}_{k} \boldsymbol{d}_{i}^{(k)}\right\|} .
$$

We refer to this kind of mapping as normalized linear encoder mapping. Then each codeword $\boldsymbol{c}_{i}^{(k)}$ of user $k$ belongs to the column space $\mathcal{U}_{k}$ of $\boldsymbol{U}_{k}$. For example, when $T=3, K=2$, and $\mathcal{D}_{1}$ and $\mathcal{D}_{2}$ are both real Grassmannian constellations of cardinality 4 , a geometric interpretation for the precoders $\boldsymbol{U}_{1}=\left[\begin{array}{ll}\boldsymbol{e}_{1} & \boldsymbol{e}_{3}\end{array}\right]$ and $\boldsymbol{U}_{2}=\left[\begin{array}{ll}\boldsymbol{e}_{2} & \boldsymbol{e}_{3}\end{array}\right]$ is provided in Fig. 1 . This geometric separation is the discrimination factor of the signals transmitted from different users.

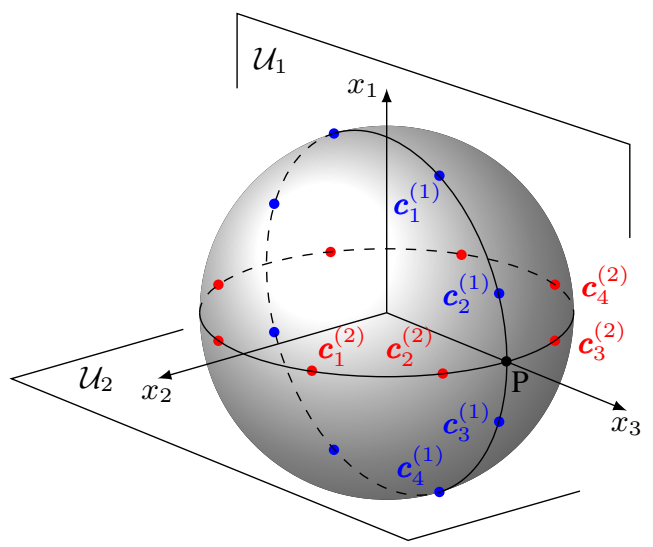

Fig. 1. A geometric interpretation on the real Grassmannian for the precoders $\boldsymbol{U}_{1}=\left[\begin{array}{ll}\boldsymbol{e}_{1} & \boldsymbol{e}_{3}\end{array}\right]$ and $\boldsymbol{U}_{2}=\left[\begin{array}{ll}\boldsymbol{e}_{2} & \boldsymbol{e}_{3}\end{array}\right]$ for $T=3, K=2, B_{1}=B_{2}=2$. The codewords of user 1 and user 2 -represented by their projections on the unit sphere-belong to the column spaces $\mathcal{U}_{1}$ and $\mathcal{U}_{2}$ of $\boldsymbol{U}_{1}$ and $\boldsymbol{U}_{2}$, respectively. The axis $x_{t}, t=1,2,3$, corresponds to the $t$-th component of a codeword.

We define $\boldsymbol{V}_{k} \in \mathbb{C}^{T \times(K-1)}$ as a basis of the orthogonal subspace of $\mathcal{U}_{k}$, i.e. $\boldsymbol{V}_{k}^{\mathrm{H}} \boldsymbol{U}_{k}=\mathbf{0}, \boldsymbol{V}_{k}^{\mathrm{H}} \boldsymbol{V}_{k}=\mathbf{I}_{K-1}$.

Property 1 (Distance preservation). If $\boldsymbol{U}_{k}^{\mathrm{H}} \boldsymbol{U}_{k}=\mathbf{I}_{T-K+1}$, the chordal distance of any pair of codewords in $\mathcal{D}_{k}$ is preserved by the normalized linear encoder mappings.

Proof. For any two codewords $\boldsymbol{d}_{1}$ and $\boldsymbol{d}_{2}$ in $\mathcal{D}_{k}$, we have $d\left(\boldsymbol{U}_{k} \boldsymbol{d}_{1}, \boldsymbol{U}_{k} \boldsymbol{d}_{2}\right)=\sqrt{1-\left|\boldsymbol{d}_{2}^{\mathrm{H}} \boldsymbol{U}_{k}^{\mathrm{H}} \boldsymbol{U}_{k} \boldsymbol{d}_{1}\right|^{2}}=\sqrt{1-\left|\boldsymbol{d}_{2}^{\mathrm{H}} \boldsymbol{d}_{1}\right|^{2}}=$ $d\left(\boldsymbol{d}_{1}, \boldsymbol{d}_{2}\right)$, since $\boldsymbol{U}_{k}^{\mathrm{H}} \boldsymbol{U}_{k}=\mathbf{I}$ by assumption.

This property states that the distance spectrum of the individual codebooks $\mathcal{C}_{k}$ is the same as that of $\mathcal{D}_{k}$ if $\boldsymbol{U}_{k}^{\mathrm{H}} \boldsymbol{U}_{k}=\mathbf{I}$.

Property 2 (Identifiability condition). With normalized linear encoders, the transmitted signals can be separated and detected error-free in the noiseless case if $\operatorname{rank}(\mathbf{H})=K$ and

$$
\boldsymbol{E}_{k}:=\boldsymbol{V}_{k}^{\mathrm{H}}\left[\boldsymbol{U}_{1} \tilde{\mathbf{x}}_{1} \ldots \boldsymbol{U}_{k-1} \tilde{\mathbf{x}}_{k-1} \boldsymbol{U}_{k+1} \tilde{\mathbf{x}}_{k+1} \ldots \boldsymbol{U}_{K} \tilde{\mathbf{x}}_{K}\right]
$$


is full rank for any $k \in\{1, \ldots, K\}$ and any possible codeword association $\left\{\tilde{\mathbf{x}}_{1}, \ldots, \tilde{\mathbf{x}}_{k-1}, \tilde{\mathbf{x}}_{k+1}, \ldots, \tilde{\mathbf{x}}_{K}\right\} \in \prod_{l \neq k} \mathcal{D}_{l}$.

Proof. For any realization of $\mathbf{H}$ such that $\operatorname{rank}(\mathbf{H})=K$, there exists a matrix $\mathbf{A}_{k} \in \mathbb{C}^{M \times(M-K+1)}$ with orthogonal columns such that $\mathbf{H}_{-k}^{\top} \mathbf{A}_{k}=\mathbf{0}$, with $\mathbf{H}_{-k}:=\left[\mathbf{h}_{1} \ldots \mathbf{h}_{k-1} \mathbf{h}_{k+1} \ldots \mathbf{h}_{K}\right]$. That is, the columns of $\mathbf{A}_{k}$ span the orthogonal complement of the column space of $\mathbf{H}_{-k}^{*}$. With $\mathrm{X}$ constructed using normalized linear encoders, in the noiseless case $\mathbf{Y}=\mathbf{X} \mathbf{H}^{\top}$, we have

$$
\boldsymbol{U}_{k}^{\dagger} \mathbf{Y} \mathbf{A}_{k}=\boldsymbol{U}_{k}^{\dagger} \mathbf{X} \mathbf{H}^{\top} \mathbf{A}_{k}=\tilde{\mathbf{x}}_{k} \mathbf{h}_{k}^{\top} \mathbf{A}_{k},
$$

where $\boldsymbol{U}_{k}^{\dagger}:=\left(\boldsymbol{U}_{k}^{\mathrm{H}} \boldsymbol{U}_{k}\right)^{-1} \boldsymbol{U}_{k}^{\mathrm{H}}$. Each column of $\tilde{\mathbf{x}}_{k} \mathbf{h}_{k}^{\top} \mathbf{A}_{k}$ is a scaled version of the Grassmannian symbol $\tilde{\mathbf{x}}_{k} \in G\left(\mathbb{C}^{T-K+1}, 1\right)$, and thus is identical to $\tilde{\mathbf{x}}_{k}$ in $G\left(\mathbb{C}^{T-K+1}, 1\right)$. Therefore, $\tilde{\mathbf{x}}_{k}$ can be detected without error from $\mathbf{Y}$ if $\mathbf{A}_{k}$ is known. Note that in the noiseless case, $\mathbf{A}_{k}$ spans the null space of $\boldsymbol{V}_{k}^{\mathrm{H}} \mathbf{Y}$ since $\boldsymbol{V}_{k}^{\mathrm{H}} \mathbf{Y} \mathbf{A}_{k}=\boldsymbol{E}_{k} \mathbf{H}_{-k}^{\top} \mathbf{A}_{k}=\mathbf{0}$, and thus $\mathbf{A}_{k}$ can be identified from $\boldsymbol{V}_{k}^{\mathrm{H}} \mathbf{Y}$ without the knowledge of $\mathbf{H}$ as long as $\boldsymbol{E}_{k}$ is full rank.

\section{A. Criteria for precoder design}

If the transmitters could cooperate, the system could be seen as a $K \times M$ MIMO point-to-point non-coherent channel, for which the high-SNR optimal input is uniformly distributed on the Grassmannian $G\left(\mathbb{C}^{T}, K\right)$ of dimension $K$ [2]. Inspired by this, we view $\widehat{\mathcal{C}}:=$ $\left\{\bigoplus_{k=1}^{K} \operatorname{span}\left(\mathbf{x}_{k}\right):\left\{\mathbf{x}_{1}, \ldots, \mathbf{x}_{K}\right\} \in \prod_{k=1}^{K} \mathcal{C}_{k}\right\}$, as a Grassmannian constellation in $G\left(\mathbb{C}^{T}, K\right)$. We would like to design the precoders such that the elements of $\widehat{\mathcal{C}}$ are maximally distant from each other. However, solving the optimization over $\widehat{\mathcal{C}}$ is analytically challenging and numerically cumbersome. Thus, we consider a more greedy approach by taking the QR factorization of the precoder:

$$
\boldsymbol{U}_{k}=\boldsymbol{Q}_{k} \boldsymbol{R}_{k}
$$

where the truncated-unitary matrix $\boldsymbol{Q}_{k} \in \mathbb{C}^{T \times(T-K+1)}$ imposes the subspace $\mathcal{U}_{k}$ which the codewords $\boldsymbol{c}_{i}^{(k)}$ lie in, and the upper triangular matrix $\boldsymbol{R}_{k} \in \mathbb{C}^{(T-K+1) \times(T-K+1)}$ partially decides the orientation of the codewords in this subspace. Both $\boldsymbol{Q}_{k}$ and $\boldsymbol{R}_{k}$ need to be optimized so as to maximize the distance between elements of $\widehat{\mathcal{C}}$.

First, we would like to optimize $Q_{k}$ so that the subspaces $\mathcal{U}_{k}$ are as distant from each other as possible. Considering the max-min chordal distance optimization problem, we have

$$
\begin{aligned}
& \left\{\boldsymbol{Q}_{1}, \ldots, \boldsymbol{Q}_{K}\right\} \\
& \quad=\arg \max _{\boldsymbol{Q}_{k}: \boldsymbol{Q}_{k}^{\mathrm{H}} \boldsymbol{Q}_{k}=\mathbf{I}, k=1, \ldots, K} \min _{i, j \in\{1, \ldots, K\}}\left\|\boldsymbol{Q}_{i} \boldsymbol{Q}_{i}^{\mathrm{H}}-\boldsymbol{Q}_{j} \boldsymbol{Q}_{j}^{\mathrm{H}}\right\|_{F}^{2} \\
& =\arg \min _{\boldsymbol{Q}_{k}: \boldsymbol{Q}_{k}^{\mathrm{H}} \boldsymbol{Q}_{k}=\mathbf{m}, k=1, \ldots, K}\left\|\boldsymbol{V}_{i} \boldsymbol{V}_{i}^{\mathrm{H}}-\boldsymbol{V}_{j} \boldsymbol{V}_{j}^{\mathrm{H}}\right\|_{F}^{2},
\end{aligned}
$$

since $V_{k}$ is the orthogonal complement of $\boldsymbol{Q}_{k}, k=1, \ldots, K$. When $T \geq K(K-1)$, the solution is obvious: we set all $V_{k}$ orthogonal to each other as in the following example.

Precoder Type I. If $T \geq K(K-1)$, for $k=1, \ldots, K$,

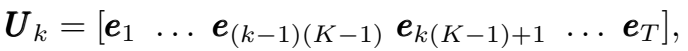

$$
\begin{aligned}
& \boldsymbol{V}_{k}=\left[\begin{array}{lll}
\boldsymbol{e}_{(k-1)(K-1)+1} & \ldots & \boldsymbol{e}_{k(K-1)}
\end{array}\right]
\end{aligned}
$$

Proposition 1. Precoder Type I fulfills the identifiability condition if $\operatorname{det}\left(\mathbf{X}_{-k,(k-1)(K-1)+1: k(K-1)}\right) \neq 0$, for any $k \in\{1, \ldots, K\}$ and any $\mathbf{X}_{-k} \in \prod_{l \neq k} \mathcal{C}_{l}$, where $\mathbf{X}_{-k,(k-1)(K-1)+1: k(K-1)}$ denotes the sub-matrix containing the rows from $(k-1)(K-1)+1$ to $k(K-1)$ of $\mathbf{X}_{-k}$.

For $\boldsymbol{R}_{k}$, we consider the special case $\boldsymbol{R}_{k}=$ $\operatorname{diag}\left(\lambda_{1}^{(k)}, \lambda_{2}^{(k)}, \ldots, \lambda_{T-K+1}^{(k)}\right)$, where the factor $\lambda_{i}^{(k)}$ indicates the power fraction in the eigenmode of $\mathcal{U}_{k}$ represented by column $i$ of $\boldsymbol{U}_{k}$. By adjusting these factors, we control the orientation of the codewords in $\mathcal{U}_{k}$. As the subspaces $\mathcal{U}_{k}, k=1, \ldots, K$, can overlap, it is favorable to allocate less power in their intersection. In Fig. 1, this can be seen as putting the points representing the codewords further away from the point $P$ representing the intersection of $\mathcal{U}_{1}$ and $\mathcal{U}_{2}$. On the other hand, considering the concatenation of precoders, the power control should not favor any particular direction. Following is an example of precoders with power control.

Precoder Type II. For $k=1, \ldots, K$,

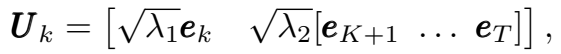

$$
\begin{aligned}
& \boldsymbol{V}_{k}=\left[\begin{array}{llllll}
\boldsymbol{e}_{1} & \ldots & \boldsymbol{e}_{k-1} & \boldsymbol{e}_{k+1} & \ldots & \boldsymbol{e}_{K}
\end{array}\right],
\end{aligned}
$$

where $\lambda_{1}=\frac{K(T-K+1)}{T}$ and $\lambda_{2}=\frac{T-K+1}{T}$ so that in $\left[\begin{array}{lll}U_{1} & \ldots & U_{K}\end{array}\right]$, equal power is allocated to every direction.

Proposition 2. Precoder Type II fulfills the identifiability condition if $\prod_{k=1}^{K} \tilde{\mathbf{x}}_{k}^{\top} \boldsymbol{e}_{1} \neq 0$ for any $\left\{\tilde{\mathbf{x}}_{1}, \ldots, \tilde{\mathbf{x}}_{K}\right\} \in \prod_{k=1}^{K} \mathcal{D}_{k}$.

We note that given a fixed $\mathcal{D}_{k}$ whose design does not necessarily take the conditions in Propositions 1 and 2 into account, we can apply a random rotation to have $\tilde{\mathcal{D}}_{k}=\mathbf{F}_{k} \mathcal{D}_{k}$, which has the same distance spectrum as $\mathcal{D}_{k}$, where $\mathbf{F}_{k} \in$ $\mathbb{C}^{(T-K+1) \times(T-K+1)}$ is a random unitary matrix.

\section{Multiple-Access Decoder Design}

In this section, we consider the problem of decoding the codewords $\tilde{\mathbf{x}}_{k} \in \mathcal{D}_{k}, k=1, \ldots, K$.

\section{A. Maximum-likelihood decoder}

The maximum-likelihood (ML) decoder is

$$
\left\{\hat{\tilde{\mathbf{x}}}_{1}, \ldots, \hat{\tilde{\mathbf{x}}}_{K}\right\}=\arg \max _{\tilde{\mathbf{x}}_{k} \in \mathcal{D}_{k}, k=1, \ldots, K} p(\mathbf{Y} \mid \mathbf{X})
$$

where $\mathbf{X}=\left[\begin{array}{lll}\mathbf{x}_{1} & \ldots & \mathbf{x}_{K}\end{array}\right]=\left[\begin{array}{llll}\boldsymbol{U}_{1} \tilde{\mathbf{x}}_{1} & \ldots & \boldsymbol{U}_{K} \tilde{\mathbf{x}}_{K}\end{array}\right]$. We remark that with respect to the ML decoder, the set of precoders $\left\{U_{1}, \ldots, U_{K}\right\}$ is equivalent to its rotated version $\left\{\boldsymbol{F} \boldsymbol{U}_{1}, \ldots, \boldsymbol{F} \boldsymbol{U}_{K}\right\}$ for any $T \times T$ unitary matrix $\boldsymbol{F}$ because $p(\mathbf{Y} \mid \mathbf{X})=p(\boldsymbol{F} \mathbf{Y} \mid \boldsymbol{F} \mathbf{X})$ for any channel distribution since the Gaussian noise is rotationally invariant.

In the case of i.i.d. Rayleigh fading, i.e. $\mathbf{h}_{k}, k=1, \ldots, K$, are independent and follow $\mathcal{C N}\left(0, \mathbf{I}_{M}\right)$, given $\mathbf{X}$, the output $\mathbf{Y}$ is a Gaussian matrix with independent columns having the same covariance matrix $\sigma^{2} \mathbf{I}_{T}+\mathbf{X} \mathbf{X}^{\mathrm{H}}$. Therefore,

$$
\begin{aligned}
& p(\mathbf{Y} \mid \mathbf{X})=\frac{\exp \left(-\operatorname{tr}\left\{\mathbf{Y}^{\mathrm{H}}\left(\sigma^{2} \mathbf{I}_{T}+\mathbf{X} \mathbf{X}^{\mathrm{H}}\right)^{-1} \mathbf{Y}\right\}\right)}{\pi^{M T} \operatorname{det}^{M}\left(\sigma^{2} \mathbf{I}_{T}+\mathbf{X} \mathbf{X}^{\mathrm{H}}\right)} \\
& =\frac{\exp \left(-\frac{1}{\sigma^{2}}\|\mathbf{Y}\|_{F}^{2}+\frac{1}{\sigma^{2}}\left\|\mathbf{Y}^{\mathrm{H}} \mathbf{X}\left(\sigma^{2} \mathbf{I}_{K}+\mathbf{X}^{\mathrm{H}} \mathbf{X}\right)^{-\frac{1}{2}}\right\|_{F}^{2}\right)}{\pi^{M T} \operatorname{det}^{M}\left(\sigma^{2} \mathbf{I}_{K}+\mathbf{X}^{\mathrm{H}} \mathbf{X}\right)} .
\end{aligned}
$$


Thus, the ML decoder is

$$
\begin{array}{r}
\left\{\hat{\tilde{\mathbf{x}}}_{1}, \ldots, \hat{\tilde{\mathbf{x}}}_{K}\right\}=\arg \max _{\tilde{\mathbf{x}}_{k} \in \mathcal{D}_{k}, k=1, \ldots, K}\left(\left\|\mathbf{Y}^{\mathrm{H}} \mathbf{X}\left(\sigma^{2} \mathbf{I}_{K}+\mathbf{X}^{\mathrm{H}} \mathbf{X}\right)^{-\frac{1}{2}}\right\|_{F}^{2}\right. \\
\left.-M \sigma^{2} \log \operatorname{det}\left(\sigma^{2} \mathbf{I}_{K}+\mathbf{X}^{\mathrm{H}} \mathbf{X}\right)\right) .
\end{array}
$$

Note that if $\mathbf{x}_{1}, \ldots, \mathbf{x}_{K}$ are orthonormal such that $\mathbf{X}^{\natural} \mathbf{X}=\mathbf{I}_{K}$ for any $\mathbf{x}_{1}, \ldots, \mathbf{x}_{K}$ in the corresponding codebook (which is true when $K=1$ ), then the ML decoder reduces to

$$
\left\{\hat{\tilde{\mathbf{x}}}_{1}, \ldots, \hat{\tilde{\mathbf{x}}}_{K}\right\}=\arg \max _{\tilde{\mathbf{x}}_{k} \in \mathcal{D}_{k}, k=1, \ldots, K}\left\|\mathbf{Y}^{\boldsymbol{H}} \mathbf{X}\right\|_{F}^{2},
$$

which coincides with the ML decoder for the single-user MIMO channel [3, Sec. V]. However, since the users's symbols are generated independently, it is not true in general that $\mathbf{X}^{\mathbf{H}} \mathbf{X}=\mathbf{I}_{K}$ for all possible codeword associations.

Implementing the ML decoder (16) requires enumerating over $\prod_{k=1}^{K} \mathcal{D}_{k}$, which has complexity $O\left(2^{B K}\right)$. Thus, we propose a greedy approach in the following.

\section{B. Low-complexity greedy decoders}

The greedy approach exploits the structure of the precoders to separate the signals of different users and decode each signal as in the single-user case. As a consequence, the multi-user ML detection is broken down into $K$ single-user detection problems of total complexity $O\left(K 2^{B}\right)$, or even lower if $\mathcal{D}_{k}$ has a structure. The details are as follows.

1) Separation-first decoder: This decoder exploits Property 2 . We directly separate the signal of user $k$ using a matrix $\mathbf{A}_{k} \in \mathbb{C}^{M \times(M-K+1)}, \mathbf{A}_{k}^{\mathrm{H}} \mathbf{A}_{k}=\mathbf{I}_{M-K+1}$, computed from $\mathbf{Y}$ such that $\boldsymbol{V}_{k}^{\mathrm{H}} \mathbf{Y} \mathbf{A}_{k}=\mathbf{0}$. The matrix $\mathbf{A}_{k}$ spans the null-space of $\boldsymbol{V}_{k}^{\mathrm{H}} \mathbf{Y}=\boldsymbol{E}_{k} \mathbf{H}_{-k}+\boldsymbol{V}_{k}^{\mathrm{H}} \mathbf{W}$ and serves as an estimate of the basis of the subspace orthogonal to the row space of $\mathbf{H}_{-k}^{*}$. Then,

$$
\tilde{\mathbf{Y}}_{k}=\boldsymbol{U}_{k}^{\dagger} \mathbf{Y} \mathbf{A}_{k}=\tilde{\mathbf{x}}_{k} \mathbf{h}_{k}^{\top} \mathbf{A}_{k}+\boldsymbol{U}_{k}^{\dagger}\left(\sum_{l=1, l \neq k}^{K} \boldsymbol{U}_{l} \tilde{\mathbf{x}}_{l} \mathbf{h}_{l}^{\top}+\mathbf{W}\right) \mathbf{A}_{k}
$$

is equivalent to a SIMO point-to-point channel output with channel vector $\tilde{\mathbf{h}}_{k}=\mathbf{A}_{k}^{\top} \mathbf{h}_{k} \in \mathbb{C}^{M-K+1}$ and equivalent noise $\tilde{\mathbf{W}}_{k}=\boldsymbol{U}_{k}^{\dagger}\left(\sum_{l=1, l \neq k}^{K} \boldsymbol{U}_{l} \tilde{\mathbf{x}}_{l} \mathbf{h}_{l}^{\top}+\mathbf{W}\right) \mathbf{A}_{k}$. We next decode $\tilde{\mathbf{x}}_{k}$ from $\tilde{\mathbf{Y}}_{k}$ using a single-user decoder.

By construction, $\mathbf{A}_{k}$ is a truncated unitary matrix independent of $\mathbf{h}_{k}$. Thus, in the case of Rayleigh fading $\mathbf{h}_{k} \sim \mathcal{C N}\left(0, \mathbf{I}_{M}\right)$, we get $\tilde{\mathbf{h}}_{k} \sim \mathcal{C N}\left(0, \mathbf{I}_{M-K+1}\right)$ which allows to approximate the ML decoder for $\tilde{\mathbf{x}}_{k}$, considering the equivalent noise as a white Gaussian vector, by

$$
\hat{\tilde{\mathbf{x}}}_{k}=\arg \max _{\tilde{\mathbf{x}} \in \mathcal{D}_{k}}\left\|\tilde{\mathbf{Y}}_{k}^{\mathrm{H}} \tilde{\mathbf{x}}\right\|^{2} .
$$

In general, the above operation has complexity $O\left(2^{B}\right)$ per user; note that this complexity can be further reduced if the single-user codebook $\mathcal{D}_{k}$ has a structure [6], [7], [8].

2) Denoising-first decoder: This decoder is similar to the separation-first decoder except that before the separation step (find $\mathbf{A}_{k}$ and compute $\tilde{\mathbf{Y}}_{k}$ ), we perform a denoising step by solving

$$
\{\hat{\mathbf{X}}, \mathbf{H}\}=\arg \max _{\mathbf{H} \in \mathbb{C}^{M \times K}, \mathbf{X} \in \mathbb{C}^{T \times K}: \mathbf{X}^{H} \mathbf{X}=\mathbf{I}}\left\|\mathbf{Y}-\mathbf{X H}^{\top}\right\|_{F}^{2} .
$$

The solution of this optimization is $\mathbf{H}=\mathbf{Y}^{\top} \hat{\mathbf{X}}^{*}\left(\hat{\mathbf{X}}^{\top} \hat{\mathbf{X}}^{*}\right)^{-1}$ with $\hat{\mathbf{X}}=\arg \max _{\mathbf{X} \in \mathbb{C}^{T \times K}: \mathbf{X}^{\mathrm{H}} \mathbf{X}=\mathbf{I}}\left\|\mathbf{Y}^{\boldsymbol{H}} \mathbf{X}\right\|_{F}^{2}$. Thus $\hat{\mathbf{X}}$ is the collection of the $K$ singular vectors of $\mathbf{Y}$ associated to the $K$ strongest singular values. Then we find the unique $\hat{\tilde{\mathbf{x}}}_{1}, \ldots, \hat{\tilde{\mathbf{x}}}_{K}$ such that

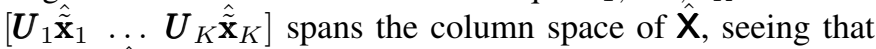
each $\boldsymbol{U}_{k} \hat{\tilde{\mathbf{x}}}_{k}$ is in the column space of $\boldsymbol{U}_{k}$. In order to do so, we now apply the separation step in separation-first decoder to $\hat{\mathbf{X}}$ That is, we first find a matrix $\mathbf{A}_{k} \in \mathbb{C}^{M \times(M-K+1)}, \mathbf{A}_{k}^{\mathrm{H}} \mathbf{A}_{k}=$ $\mathbf{I}_{M-K+1}$, such that $\boldsymbol{V}_{k}^{\mathrm{H}} \hat{\mathbf{X}} \mathbf{A}_{k}=0$, then consider $\tilde{\mathbf{Y}}_{k}=\boldsymbol{U}_{k}^{\dagger} \hat{\mathbf{X}} \mathbf{A}_{k}$ as the input of a single-user decoder.

3) Interference cancellation: In the above decoders, the interference $\sum_{l=1, l \neq k}^{K} \boldsymbol{U}_{l} \tilde{\mathbf{x}}_{l} \mathbf{h}_{l}^{\top}$ is partially canceled using an estimation $\left(\mathbf{A}_{k}\right)$ of its row space. Now, assuming that we have the estimates $\tilde{\tilde{\mathbf{x}}}_{1}, \ldots, \hat{\tilde{\mathbf{x}}}_{K}$ of $\tilde{\mathbf{x}}_{1}, \ldots, \tilde{\mathbf{x}}_{K}$ from the separationfirst or denoising-first decoder, we can try to further cancel the residual interference. Let $\mathbf{O}_{k}$ be an orthonormal basis of the column space of $\left[\boldsymbol{U}_{1} \hat{\tilde{\mathbf{x}}}_{1} \ldots \boldsymbol{U}_{k-1} \hat{\tilde{\mathbf{x}}}_{k-1} \boldsymbol{U}_{k+1} \hat{\tilde{\mathbf{x}}}_{k+1} \ldots \boldsymbol{U}_{K} \hat{\tilde{\mathbf{x}}}_{K}\right]$, which is approximately, up to the noise perturbation, the column space of the interference. We project the received signal onto the orthogonal of this approximate interference space

$$
\begin{aligned}
\tilde{\mathbf{Y}}_{k}= & \boldsymbol{U}_{k}^{\dagger}\left(\mathbf{I}_{T}-\mathbf{O}_{k} \mathbf{O}_{k}^{\mathrm{H}}\right) \mathbf{Y} \mathbf{A}_{k} \\
= & \boldsymbol{U}_{k}^{\dagger}\left(\mathbf{I}_{T}-\mathbf{O}_{k} \mathbf{O}_{k}^{\mathrm{H}}\right) \boldsymbol{U}_{k} \tilde{\mathbf{x}}_{k} \tilde{\mathbf{h}}_{k}^{\top} \\
& +\boldsymbol{U}_{k}^{\dagger}\left(\mathbf{I}_{T}-\mathbf{O}_{k} \mathbf{O}_{k}^{\mathrm{H}}\right)\left(\sum_{l=1, l \neq k}^{K} \boldsymbol{U}_{l} \tilde{\mathbf{x}}_{l} \mathbf{h}_{l}^{\top}+\mathbf{W}\right) \mathbf{A}_{k} .
\end{aligned}
$$

We then decode for $\tilde{\mathbf{x}}_{k}$ from $\tilde{\mathbf{Y}}_{k}$ using a single-user decoder. With these new estimates $\hat{\tilde{\mathbf{x}}}_{1}, \ldots, \hat{\tilde{\mathbf{x}}}_{K}$, we can repeat the interference cancellation, making it an iterative process. Each iteration is equivalent in complexity to the separation-first or denoising-first decoder.

\section{Performance Evaluation}

We evaluate the performance of our proposed scheme with two types of precoders in Section III-A for a system of $K=3$ users and $M=8$ receive antennas over Rayleigh block-fading channel with coherence time $T=6$ symbols. We choose $\mathcal{D}_{1}=$ $\ldots=\mathcal{D}_{K}$ as the structured codebook proposed in [8]. For Precoder Type I, we further apply a random rotation on each of these codebooks. We compare our scheme with time division multiple access (TDMA) and a pilot-based scheme for the same data rate. In TDMA, each user is allocated a coherence block in a round-robin manner and also uses the codebook in [8]. In the pilot-based scheme, the users send orthogonal pilots in the first $K$ time slots and data in the last $T-K$ slots; the receiver uses minimum mean square error (MMSE) channel estimation followed by a MMSE equalizer based on the channel estimates.

First, we show the symbol (codeword) error rate and bit error rate (with Gray mapping) in Fig. 2. We can see that with Precoder Type II, the separation-first and denoising-first decoders are on par with the pilot-based scheme and better than TDMA. The performance of our scheme is further improved with interference cancellation (one iteration) and it outperforms the pilot-based scheme by $1.5 \mathrm{~dB}$ for a bit error rate of $10^{-4}$. 


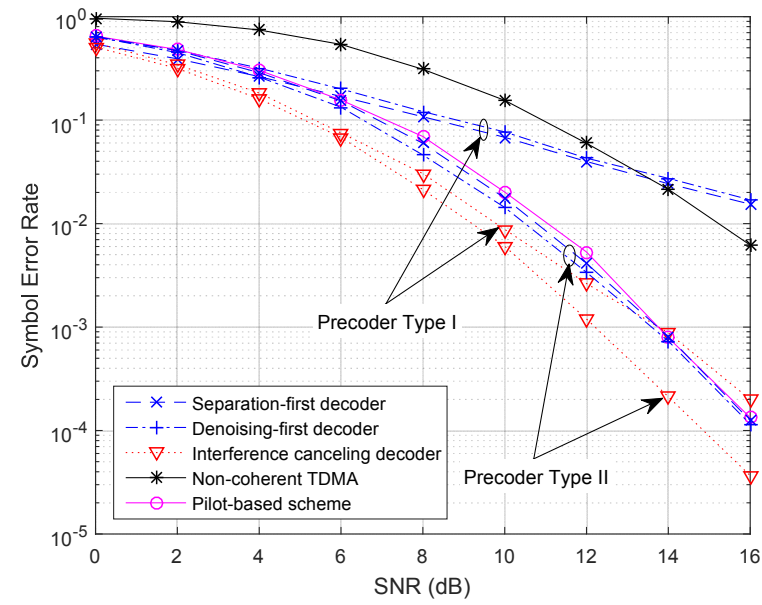

(a) Symbol error rate

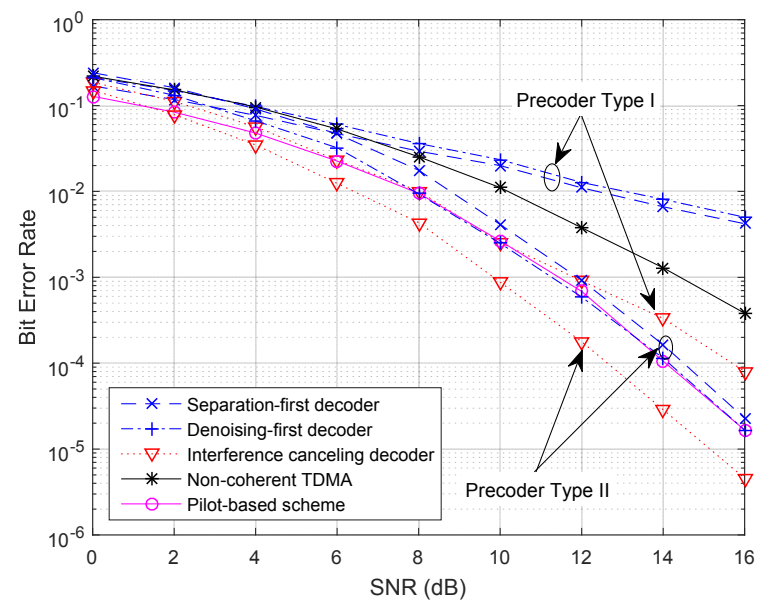

(b) Bit error rate

Fig. 2. Error rates of the proposed multiple access scheme in comparison with TDMA and pilot-based scheme for $T=6, M=8, K=3$, and data rate of 8 bits/codeword/user.

Next, we integrate a systematic parallel concatenated rate- $1 / 3$ LTE turbo code. The turbo encoder takes in each packet of 1008 bits; the turbo decoder calculates the log-likelihood ratio of the received encoded bits and performs 10 iterations of decoding. The bit error rate with this turbo code is presented in Fig. 3. Our scheme with Precoder Type II and one iteration of interference cancellation in the decoder achieves the best performance and outperforms the pilot-based scheme. Interestingly, although Precoder Type I is worse than Precoder Type II when there is no channel code, it becomes better, with separation-first decoder or denoising-first decoder, when turbo code is employed.

\section{CONCLUSION}

We have proposed a multiple access scheme for non-coherent SIMO communications. We define for each user a unique encoder mapping for codebook generation. In particular, with normalized linear encoder mappings, we introduce a geometric separation amongst the users's codebooks. Exploiting this geometric separation, we propose greedy decoders that greatly reduce the complexity with respect to the maximum likelihood

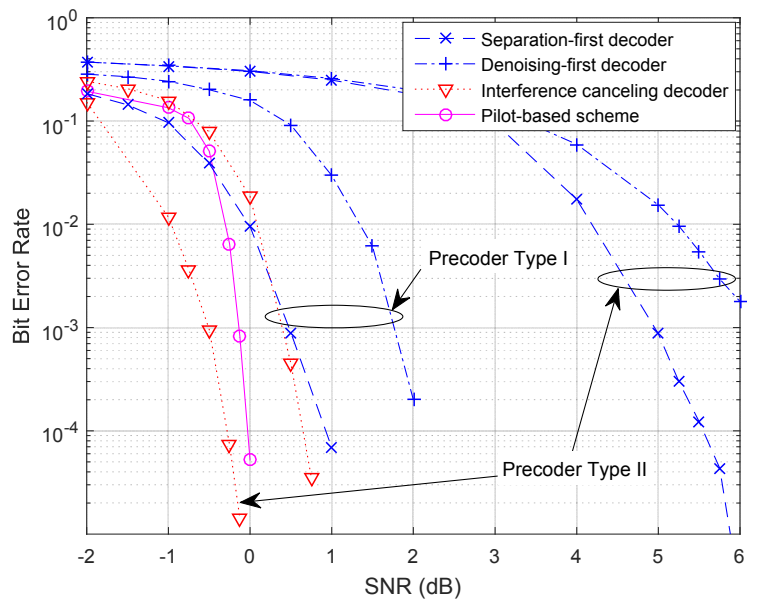

Fig. 3. Bit error rate of the proposed multiple access scheme in comparison with TDMA and pilot-based scheme with turbo codes for $T=6, M=8, K=3$, and data rate of 8 bits/codeword/user.

decoder while achieving better performance than time division multiple access and the coherent approach based on pilot.

\section{REFERENCES}

[1] B. Hassibi and B. M. Hochwald, "How much training is needed in multiple-antenna wireless links?" IEEE Trans. Inf. Theory, vol. 49, no. 4 pp. 951-963, Apr. 2003.

[2] L. Zheng and D. N. C. Tse, "Communication on the Grassmann manifold: A geometric approach to the noncoherent multiple-antenna channel," IEEE Trans. Inf. Theory, vol. 48, no. 2, pp. 359-383, Feb. 2002.

[3] B. M. Hochwald and T. L. Marzetta, "Unitary space-time modulation for multiple-antenna communications in Rayleigh flat fading," IEEE Trans. Inf. Theory, vol. 46, no. 2, pp. 543-564, Mar. 2000.

[4] D. Agrawal, T. J. Richardson, and R. L. Urbanke, "Multiple-antenna signal constellations for fading channels," IEEE Trans. Inf. Theory, vol. 47 , no. 6, pp. 2618-2626, Sep. 2001.

[5] R. H. Gohary and T. N. Davidson, "Noncoherent MIMO communication: Grassmannian constellations and efficient detection," IEEE Trans. Inf. Theory, vol. 55, no. 3, pp. 1176-1205, Mar. 2009.

[6] B. M. Hochwald, T. L. Marzetta, T. J. Richardson, W. Sweldens, and R. Urbanke, "Systematic design of unitary space-time constellations," IEEE Trans. Inf. Theory, vol. 46, no. 6, pp. 1962-1973, Sep. 2000.

[7] I. Kammoun, A. M. Cipriano, and J. C. Belfiore, "Non-coherent codes over the Grassmannian," IEEE Trans. Wireless Commun., vol. 6, no. 10, pp. 3657-3667, Oct. 2007.

[8] K. H. Ngo, A. Decurninge, M. Guillaud, and S. Yang, "Design and analysis of a practical codebook for non-coherent communications," in Asilomar Conference on Signals, Systems, and Computers, Oct 2017, pp. 1237-1241.

[9] S. Murugesan, E. Uysal-Biyikoglu, and P. Schniter, "Optimization of training and scheduling in the non-coherent SIMO multiple access channel," IEEE J. Sel. Areas Commun., vol. 25, no. 7, pp. 1446-1456, Sep. 2007.

[10] K.-H. Ngo, S. Yang, and M. Guillaud, "The optimal DoF region for the two-user non-coherent SIMO multiple-access channel," in IEEE Information Theory Workshop (ITW), Guangzhou, China, Nov. 2018.

[11] M. Fadel and A. Nosratinia, "Coherence disparity in broadcast and multiple access channels," IEEE Trans. Inf. Theory, vol. 62, no. 12, pp. 7383-7401, Dec. 2016.

[12] A. Manolakos, M. Chowdhury, and A. Goldsmith, "Energy-based modulation for noncoherent massive SIMO systems," IEEE Transactions on Wireless Communications, vol. 15, no. 11, pp. 7831-7846, Nov 2016

[13] V. M. Baeza, A. G. Armada, W. Zhang, M. El-Hajjar, and L. Hanzo, "A noncoherent multiuser large-scale SIMO system relying on M-ary DPSK and BICM-ID," IEEE Trans. Veh. Technol., vol. 67, no. 2, pp. 1809-1814, Feb 2018.

[14] A. Schenk and R. F. H. Fischer, "Noncoherent detection in massive MIMO systems," in International ITG Workshop on Smart Antennas, Mar. 2013, pp. 1-8. 